ARTICLE

\title{
Understanding catalysis in a multiphasic two-dimensional transition metal dichalcogenide
}

\author{
Stanley S. Chou' ${ }^{1}$ Na Sai ${ }^{2}$, Ping Lü ${ }^{3}$, Eric N. Coker ${ }^{1}$, Sheng Liu ${ }^{4}$, Kateryna Artyushkova ${ }^{5}$, Ting S. Luk ${ }^{4}$, \\ Bryan Kaehr ${ }^{1,5} \&$ C. Jeffrey Brinker ${ }^{1,4,5}$
}

Establishing processing-structure-property relationships for monolayer materials is crucial for a range of applications spanning optics, catalysis, electronics and energy. Presently, for molybdenum disulfide, a promising catalyst for artificial photosynthesis, considerable debate surrounds the structure/property relationships of its various allotropes. Here we unambiguously solve the structure of molybdenum disulfide monolayers using high-resolution transmission electron microscopy supported by density functional theory and show lithium intercalation to direct a preferential transformation of the basal plane from $2 \mathrm{H}$ (trigonal prismatic) to $1 \mathrm{~T}^{\prime}$ (clustered Mo). These changes alter the energetics of molybdenum disulfide interactions with hydrogen $\left(\Delta G_{H}\right)$, and, with respect to catalysis, the $1 T^{\prime}$ transformation renders the normally inert basal plane amenable towards hydrogen adsorption and hydrogen evolution. Indeed, we show basal plane activation of $1 T^{\prime}$ molybdenum disulfide and a lowering of $\Delta G_{H}$ from $+1.6 \mathrm{eV}$ for $2 \mathrm{H}$ to $+0.18 \mathrm{eV}$ for $1 \mathrm{~T}^{\prime}$ ', comparable to $2 \mathrm{H}$ molybdenum disulfide edges on $\mathrm{Au}(111)$, one of the most active hydrogen evolution catalysts known.

\footnotetext{
${ }^{1}$ Advanced Materials Laboratory, Sandia National Laboratories, Albuquerque, New Mexico 87106, USA. ${ }^{2}$ Department of Physics, The University of Texas at Austin, Austin, Texas 78712, USA. ${ }^{3}$ Department of Materials Characterization \& Performance, Sandia National Laboratories, Albuquerque, New Mexico 87123, USA. ${ }^{4}$ Center For Integrated Nanotechnologies (CINT), Sandia National Laboratories, Albuquerque, New Mexico 87123, USA. ${ }^{5}$ Department of Chemical and Biological Engineering, The University of New Mexico, Albuquerque, New Mexico 87131, USA. Correspondence and requests for materials should be addressed to S.S.C. (email: schou@sandia.gov) or to C.J.B. (email: cjbrink@sandia.gov).
} 
mproving the capacity and efficiency of the Hydrogen Evolution Reaction (HER) is an enduring challenge of green energy production and artificial photosynthesis ${ }^{1,2}$. Still, while HER in organisms evolved with time and increasing complexity, artificial HER aims to replicate the same with minimalism and simplicity. A cornerstone of the challenge is to mimic the function of natural hydrogenase enzymes, which catalyse HER in living systems. Indeed, it can be seen that without a catalyst like hydrogenase, HER does not proceed with the speed required for practical applications ${ }^{3,4}$. However, hydrogenases can be difficult to extract and purify, and can denature under non-natural operations ${ }^{5}$, consequently, inorganic alternatives are used for most applications. The most common example of this is platinum (Pt), which has served as the benchmark catalyst for HER due to its high catalytic efficiency ${ }^{6-8}$. Nevertheless, because of the scarcity and cost of $\mathrm{Pt}$, a more abundant alternative is needed for cost-effective implementation.

For this, $\mathrm{MoS}_{2}$, an earth abundant lamellar solid, has shown prominent HER catalysis nearing the efficiency of platinum ${ }^{9,10}$. However, experiments using $\mathrm{MoS}_{2}$ grown on $\mathrm{Au}(111)$ indicated that this material is only catalytic on its edge sites ${ }^{9}$. Theoretical studies corroborated these results with the Gibbs free energy of hydrogen adsorption $\left(\Delta G_{\mathrm{H}}\right)$, a measure of HER efficiency, to be feasible for catalysis only at $\mathrm{MoS}_{2}$ edges ${ }^{11,12}$; the basal plane of $\mathrm{MoS}_{2}$ does not appear to participate in catalysis, meaning the bulk of material is catalytically inert. Consequently, the maximization of $\mathrm{MoS}_{2}$ edges and mimicry of the edge structure has become a significant topic ${ }^{13-16}$.

Interestingly, recent studies have begun to show enhancement of $\mathrm{MoS}_{2}$ catalytic efficiency following lithium intercalation and exfoliation ${ }^{17-19}$. The Tafel-slope of these sheets, a benchmark of electrochemical efficiency against applied overpotential, is nearly twice that of natural $\mathrm{MoS}_{2}$ after lithium treatment ${ }^{17-19}$. As the lithium-exfoliation reactions increase the availability of basal plane surfaces but not edges, the catalytic improvements are postulated to be basal plane related. Nonetheless, because the basal plane structures of lithium-exfoliated monolayers, and indeed, many two-dimensional sheets, are difficult to characterize $\mathrm{e}^{20-22}$, the post lithiation and exfoliation structure of $\mathrm{MoS}_{2}$ remains nebulous and historically controversial, rendering the origin of this catalytic enhancement correlatively vague. To briefly recount a history of the structural understanding of $\mathrm{MoS}_{2}$ exfoliated with the assistance of lithium, it can be seen that a structural change in Li-intercalated $\mathrm{MoS}_{2}$ has been reported as far back as 1973 (ref. 23), nevertheless, interpretations of the final structure vary significantly in the literature. For example, in 1973, Somoano et al. ${ }^{23}$, observed extensive layer displacements after intercalation, suggesting the resulting product is a mixture of disordered compounds. However, in 1983, Py et al. ${ }^{24}$ reported the alkali metal exfoliated structure to be crystalline, with a first order phase transition from the natural trigonal prismatic $(2 \mathrm{H})$ to an octahedral (1T) phase. In 1989, a significant distortion to a $2 \mathrm{a}_{\mathrm{o}} \times 2 \mathrm{a}_{\mathrm{o}}$ lattice was reported by Chrissafis et al. ${ }^{25}$ In 1991, an octahedral structure was observed by Jimenez et al. and Qin et al., but a small distortion was noted, making the final crystal a $2 a_{o} \times a_{o}$ super lattice 26,27 . Atomic force microscopy images obtained in 1993, by Schumacher et al., though, indicated no distortion or superlattice ${ }^{28}$. In 1998, Dungey et al. observed a $2 \mathrm{a}_{\mathrm{o}} \times 2 \mathrm{a}_{\mathrm{o}}$ lattice with trigonal Mo clustering ${ }^{29}$, but in 1999 , a $2 a_{o} \times a_{o}$ superlattice was seen by Heising et al., with severe distortion and formation of infinite Mo-zig-zag chains ${ }^{30}$. A chain-of-diamonds motif was suggested by Petkov et al. in 2002 (ref. 31), but most recently, in 2013, Maitra et al. reported a distortion-free octahedral phase ${ }^{32}$. Chhowalla and coworkers observed similar variations, from a perfect octahedral phase with natural $2 \mathrm{H} \mathrm{MoS}_{2}$ domains ${ }^{33}$, distrted phases ${ }^{19}$, coexistence of various phases ${ }^{34}$, and also, a perfect octahedral phase ${ }^{35}$. Surprisingly, we note that the changed structures are almost always referred to as $1 \mathrm{~T}$, regardless of polymorphic structural differences. It is therefore not surprising to see recent catalytic improvement being attributed to varied structures, including octahedral $^{17,18,32}$, and distorted Mo phases ${ }^{36,37}$. Part of the confusion is likely a simple consequence of incomplete transformation resulting in identification of partially transformed and transitional states due to variations between batches, however, there may be underlying issues of stability as well, making the resultant structure, and indeed, structural based predictions of its catalytic effectiveness difficult to handle ${ }^{38}$.

To address these long-standing issues and shed light on the catalytic origin of the transformed crystals, here we take a combined experimental/theoretical approach using controlled processing conditions to achieve stable phases and density functional theory calculations to investigate the stability of these polymorphs. With the solution processing advantages of these materials ${ }^{39-41}$, we report the catalytic efficiency of HER in homogenous reactions, with experimental $\mathrm{H}_{2}$ yield of these phases, and corroborate them with calculated $\Delta G_{\mathrm{H}}$. The analysis was then extended from $\mathrm{MoS}_{2}$ to $\mathrm{WS}_{2}$ to show similarly distorted crystal phases and basal plane catalytic activation.

\section{Results}

Exfoliation and phase transformation of $\mathbf{M o S}_{2}$. A typical lithium-exfoliation reaction consisted of immersing $\mathrm{MoS}_{2}$ powder in $n$-butyl lithium $(1 \mathrm{M})$ for $72 \mathrm{~h}$ at room temperature ${ }^{31,33,42}$. The intercalated compound was then transferred to water and sonicated to yield exfoliated monolayers. After purification using centrifugation and dialysis, concentrations and purity of each batch were measured using Flame Atomic Absorption Spectrophotometry. Samples were then visualized using abberation-corrected scanning transmission electron microscopy.

As seen in Fig. 1, a typical sheet exfoliated using this method indeed yields a mixed phase (shown without false colouring in Supplementary Fig. 1). First, a trigonal prismatic $(2 \mathrm{H})$ phase that corresponds with an untransformed basal plane can be seen with symmetrical Mo-Mo spacing of $2.98 \pm 0.05 \AA$. Second, an octahedral phase with displaced sulfur atoms and symmetrical Mo arrangements $(2.95 \pm 0.06 \AA)$ is seen, albeit in small quantities. Last, large swatches of a visually distinct, tertiary phase is also present, with Mo atoms asymmetrically clustered to form one-dimensional lines of alternating light-and dark stripes. In this phase, Mo-Mo distances of $3.55 \pm 0.16 \AA$ and $2.92 \pm 0.16 \AA$ were measured. On the basis of the above, we thus conclude that within one individual sheet, a microcosm of various phases are present, with each representing differing interpretations in previous work. Nevertheless, as the stability of each phase should be an intrinsic consequence of the energetics of distinct atomic arrangements, we sought to understand the relative stabilities of each phase. Because it was previously reported that materials revert back to the natural $2 \mathrm{H}$ phase ${ }^{42}$, we reasoned that the $2 \mathrm{H}$ is the overall energetic minimum, with the other polymorphs occupying differing metastable points in relation to the $2 \mathrm{H}$.

To better define this, we employed Density Functional Theory (DFT) to independently predict the structures of possible polymorphs (see Supplementary Information for computational details). As can be seen in Table 1, a total of four polymorphs were predicted based on structural optimization of unit cells. The first two, consisting of $1 \times 1$ unit cells, were the $2 \mathrm{H}$ trigonal prismatic phase and the $1 \mathrm{~T}$ octahedral phase. In addition, a distorted phase with zig-zag chains consisting of a $2 \times 1$ supercell 


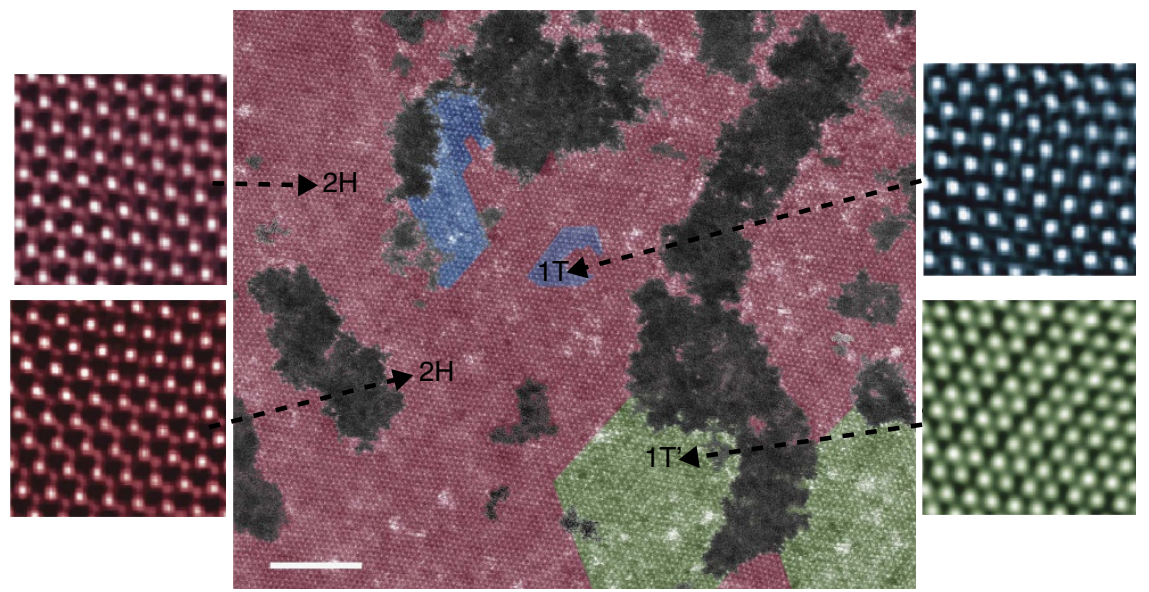

Figure 1 | Polymorphism and incomplete transformations. Polymorphisms within the basal plane exhibit coexistence of three different phases, $2 \mathrm{H}$ (trigonal prismatic, false coloured as red), $1 \mathrm{~T}$ (octahedral, false coloured as blue) and $1 \mathrm{~T}^{\prime}$ (clustered Mo, false coloured as green). Scale bar, $5 \mathrm{~nm}$.

Table 1 | Structural polymorphs of $\mathrm{MoS}_{2}$ and summary of parameters as predicted by Density Functional Theory.

$2 \mathrm{H}$

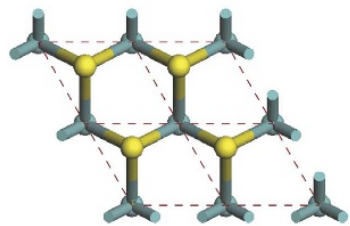

$1 \mathrm{~T}$
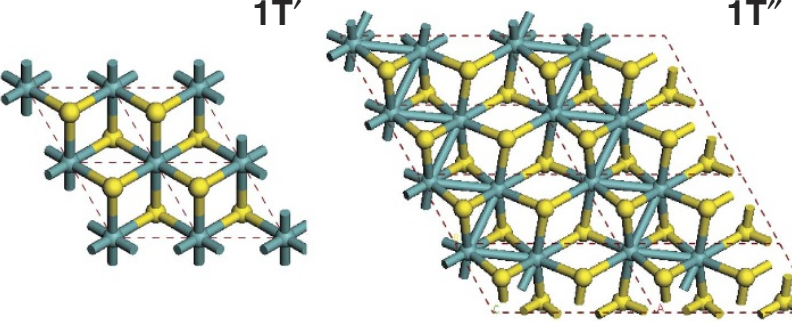

$\mathrm{T}^{\prime \prime}$

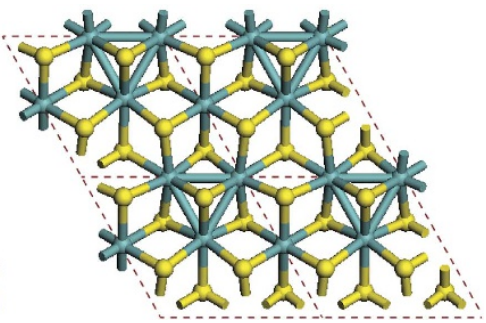

\begin{tabular}{|c|c|c|c|c|c|c|}
\hline Phase & Relative stability (eV f.u. ${ }^{-1}$ ) & a ( $(\AA)$ & b (A) & $\alpha\left({ }^{\circ}\right)$ & Mo-Mo (Å) & $E_{\mathrm{g}}(\mathrm{eV})$ \\
\hline $2 \mathrm{H}$ & 0 & 3.18 & 3.18 & 120 & 3.18 & 1.7 \\
\hline $1 \mathrm{~T}$ & 0.82 & 3.17 & 3.17 & 120 & 3.17 & Metallic \\
\hline $1 T^{\prime}$ & 0.55 & 6.55 & 3.18 & 119 & $2.77,3.18$ & 0.01 (PBE) 0.26 (HSE06) \\
\hline $1 T^{\prime \prime}$ & 0.63 & 6.44 & & 119.5 & $2.77,3.26$ & 0.1 (PBE) 0.01 (HSEO6) \\
\hline
\end{tabular}

(Top) Mo atoms are represented as teal spheres, $\mathrm{S}$ atoms as gold spheres. (Bottom) From left to right, relative stability in eV, lattice parameters a, b and $\alpha, \mathrm{Mo}-\mathrm{Mo}$ distance, and band gap $E_{\mathrm{g}}$ (eV) based on Perdew-Burke-Ernzerhof (PBE) and Heyd-Scuseria-Ernzerhof (HSE) exchange-correlation functionals.

and a phase with Mo-Mo atoms clustered into trimerized pockets in a $2 \times 2$ supercell were predicted. We dub the two later phases, $1 \mathrm{~T}^{\prime}$ and $1 \mathrm{~T}^{\prime \prime}$, respectively.

Next, the stabilities of each DFT polymorph were calculated. It can be seen that the symmetrical $1 \mathrm{~T}$ representation has the least stable ground-state $(+0.82 \mathrm{eV}$ versus $2 \mathrm{H})$. Indeed, in a $2 \times 2$ or $4 \times 4$ supercell, we find the $1 \mathrm{~T}$ phase eventually relaxes into $1 \mathrm{~T}^{\prime \prime}$, which is second in stability, at $+0.63 \mathrm{eV}$, conforming with the dynamical phonon instability previously predicted in the $1 \mathrm{~T}$ phase ${ }^{38}$. Nevertheless, the $1 \mathrm{~T}^{\prime}$ phase was significantly more stable than $1 \mathrm{~T}$ and $1 \mathrm{~T}^{\prime \prime}(+0.55 \mathrm{eV}$ versus $2 \mathrm{H})$, with a barrier against $2 \mathrm{H}$ reversion of $0.73 \mathrm{eV}$ f.u. ${ }^{-1}$ (formula unit, Supplementary Fig. 2, Supplementary Table 1). By the DFT calculations, the transformed portions within a sample, if allowed to reach metaequilibria, should therefore preferentially form $1 \mathrm{~T}^{\prime}$ instead of the other polymorphs.

To investigate these results experimentally, we prepared samples to induce equilibria by extending the lithium intercalation period. As seen in Fig. $2 \mathrm{a}$, a preferential transformation to $1 \mathrm{~T}^{\prime}$ is possible on the entirety of the basal plane. This therefore suggests that the various polymorphs observed previously are likely consequences of incomplete reactions, such as partial intercalation, with parts of the basal plane yet to undergo sufficient conversion to the $1 \mathrm{~T}^{\prime}$. Indeed, we have observed that when intercalation times are extended from 72 to $240 \mathrm{~h}$, a complete transformation to the $1 \mathrm{~T}^{\prime}$ can be achieved (Supplementary Fig. 3).

Given the DFT prediction of $1 \mathrm{~T}^{\prime}$ as a metastable phase, we examined the ability to covert $1 \mathrm{~T}^{\prime}$ back to $2 \mathrm{H}$ under electron beamillumination. As shown in Fig. 2c, sequential frames taken at $60 \mathrm{~s}$ intervals (acquired at $40 \mathrm{~s} / \mathrm{frame}$ at an electron dose rate of 2,800 electron $\AA^{-2} s^{-1}$ ) show gradual relaxation of Mo atoms from $1 \mathrm{~T}^{\prime}$ lines to symmetrical $2 \mathrm{H}$ spacing. A detailed investigation of the structures again showed the $1 \mathrm{~T}^{\prime}$ phase with Mo spacings of $3.55 \AA$ and $2.92 \AA$, and the relaxed structure displayed trigonal prismatically coordinated sulfur atoms with Mo-Mo spacing of $2.95 \AA$, in agreement with the $2 \mathrm{H}$ structure (Fig. $2 \mathrm{~d}$ ). These results corroborate the $1 \mathrm{~T}^{\prime}$ as the energetically preferred metastable phase that will relax back to $2 \mathrm{H}$ when energetic input, for example, heat, exceeds that of the metastable barrier energy. It further suggests that 

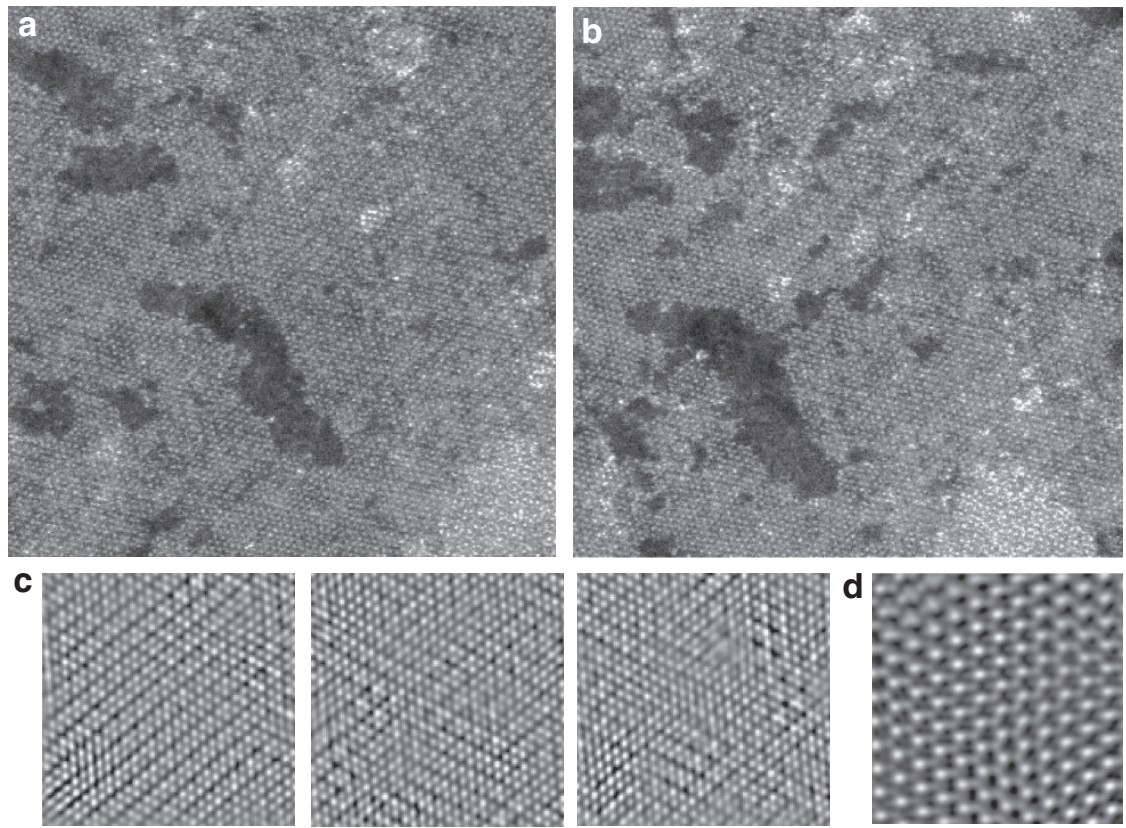

Figure 2 | Basal plane transformations. (a) $\mathrm{MoS}_{2}$ basal plane showing preferential transformation to $1 \mathrm{~T}^{\prime}$ with increased lithiation, in accordance with the calculated relative stability of the metastable phases. (b) On electron beam illumination, the basal plane exhibits a restoration to a symmetrical Mo arrangement indicative of $2 \mathrm{H}$ reversion. (c) Sequential frames taken $60 \mathrm{~s}$ apart, showing phase evolution under the electron beam. Electron dose rate was 2,800 e $\AA^{-2} \mathrm{~s}^{-1}$, with each frame requiring $40 \mathrm{~s}$ to capture. (d) An electron dosed region with sulfur atoms resolved, demonstrating $1 \mathrm{~T}^{\prime}$ to $2 \mathrm{H}$ phase reversion after illumination.
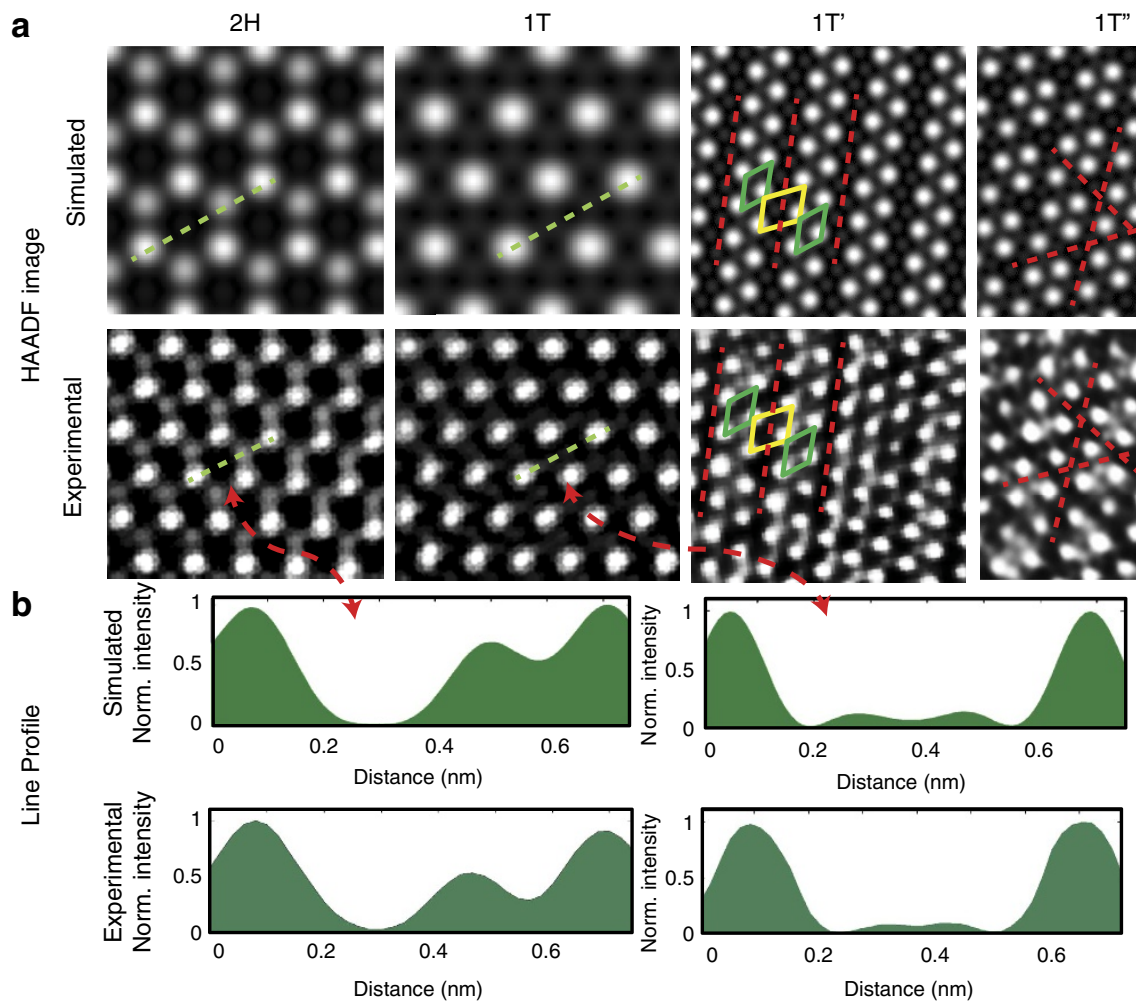

Figure 3 | Different possible phases for $\mathbf{M o S}_{\mathbf{2}}$. (a) Simulated and experimental images of different possible phases for MoS 2 . FFT mask filters have been employed for clarity. (b) line scan comparisons between $2 \mathrm{H}$ and $1 \mathrm{~T}$ using the simulated and experimental images, showing modulation of sulfur contrast.

the partial or incomplete phase transformations observed previously may be due to energetic conditions reverting the material to $2 \mathrm{H}$ during the characterization process. Supplementary Fig. 4 shows the final product of $1 \mathrm{~T}^{\prime}$ heated under inert atmosphere is a $2 \mathrm{H}$ lattice.
These observations support previous electrical characterizations of the material ${ }^{33,42}$ with $1 \mathrm{~T}^{\prime}$ having greatly reduced resistivity compared to $2 \mathrm{H}$ (calculated bandgap of 0.01 and $1.7 \mathrm{eV}$, respectively, shown in Table 1). 

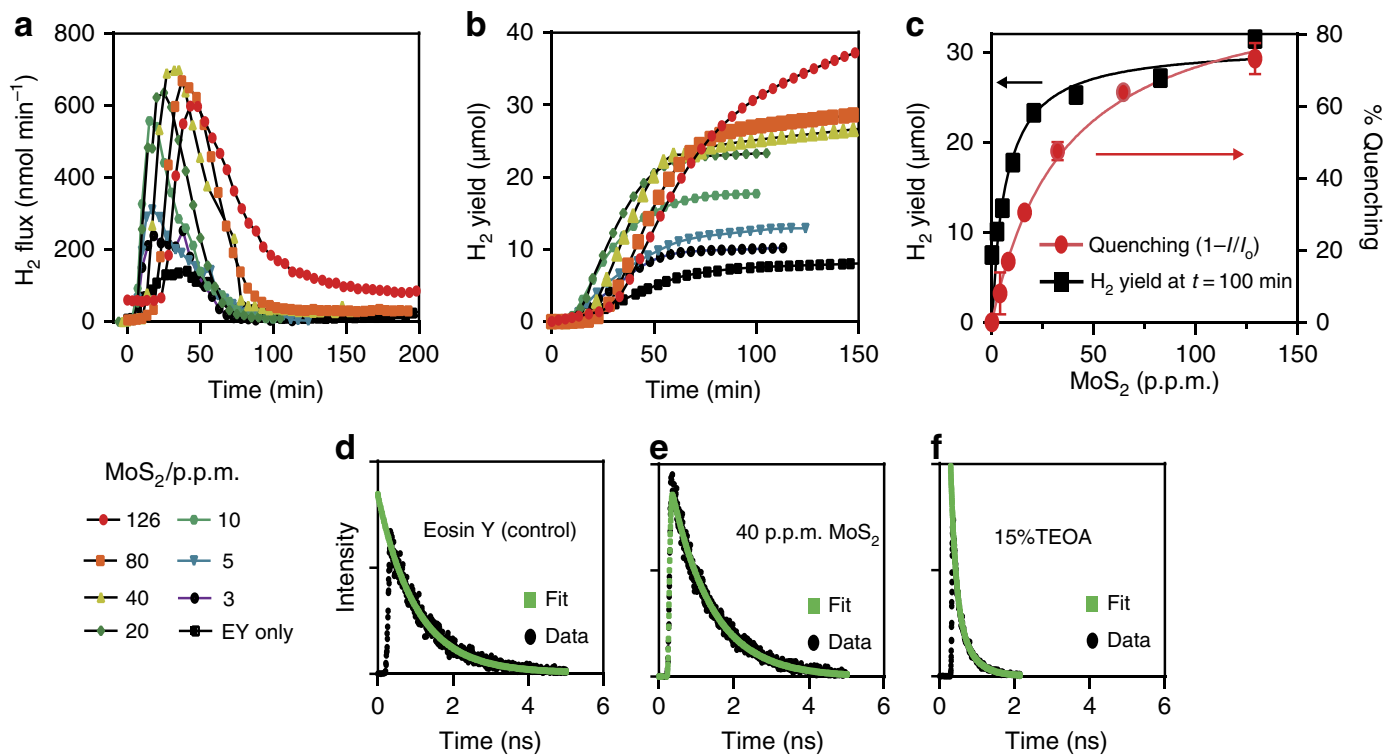

Figure 4 | Hydrogen evolution. With dye sensitization (eosin $\mathrm{Y}, \mathrm{EY}$ ), $\mathrm{MoS}_{2}$ can be used to conduct homogenous hydrogen evolution reactions in solution. (a) Real time $\mathrm{H}_{2}$ flux at incremental $\mathrm{MoS}_{2}$ concentrations. Diminished reactions are indicative of proton exhaustion, and can be restarted with acid addition. (b) Corresponding cumulative $\mathrm{H}_{2}$ yield (For $\mathbf{a}$ and $\mathbf{b}$ note key bottom left). (c) Incremental concentration of $\mathrm{MoS}_{2}$ and correlative overlay of cumulative $\mathrm{H}_{2}$ yield (black) with fluorescence quenching of dye (red) in solution. (d) fluorescence decay of eosin $\mathrm{Y}$, (e) reaction saturated with eosin $\mathrm{Y}$ and $\mathrm{MoS}_{2}$ showing similar decay, indicative of static-quenching and ground-state complex, and (f) collisional quenching between TEOA and eosin $Y$ resulting in second order decay and attenuated lifetime. Error bars denote s.d.

To corroborate the above, High-angle annular Dark-field (HAADF) images were simulated using coordinates from DFT. As seen in Fig. 3, good agreement was seen between experiment and simulations. We note in samples that are predominantly $1 \mathrm{~T}^{\prime}$ (Fig. 2a), lines modulated in two-dimensions can be seen at intersections of $1 \mathrm{~T}^{\prime}$. This may be interpreted as the $1 \mathrm{~T}^{\prime \prime}$, however, its occurrence is very localized and defective $(<5 \%)$, and consequently, not of practical significance.

Dye-sensitized HER. Following these structural insights, HER catalysis of these $\mathrm{MoS}_{2}$ polymorphs was examined. For this, we measured $\mathrm{H}_{2}$ formation using $\mathrm{MoS}_{2}$ in a homogenous photocatalysis reaction. In a typical reaction, a solution of $\mathrm{MoS}_{2}$ was sensitized with Eosin Y (EY), and Triethanolamine (TEOA) was added as a sacrificial electron donor ${ }^{43,44}$. The reaction mixture was then purged of atmospheric gasses, and irradiated with a xenon lamp tuned to 1 sun. The flow entering and exiting the reaction flask was then monitored on a gas chromatograph to quantify $\mathrm{H}_{2}$ formation inside the flask.

The reaction proceeded via photoexcitation of EY and subsequent intersystem crossing to yield a triplet excited state $\left(\mathrm{EY}^{3 *}\right)$. Acceptance of an electron via reductive quenching from the sacrificial electron donation (TEOA) then yields a radical $\mathrm{EY}^{-32,43,45-47}$. The highly reductive $\mathrm{EY}^{-}$can then reduce a proton to form $\mathrm{H}_{0}$, return the electron to an oxidized TEOA, or transfer the electron to another catalyst such as the $\mathrm{MoS}_{2}$. As seen in Fig. 4a, in the absence of $\mathrm{MoS}_{2}$, direct reduction of protons by radicalized EY occurs by collisional reductive quenching with TEOA (Supplementary Fig. 5), and generates a peak $\mathrm{H}_{2}$ flux of $150 \mathrm{nmol} \mathrm{min}{ }^{-1}$. Over the course of $100 \mathrm{~min}$, a yield of $7.5 \mu \mathrm{mols}$ was observed, giving a typical activity of $4.5 \mu \mathrm{molh}^{-1}$ (Fig. 4b). Sequential addition of $1 \mathrm{~T}^{\prime}$ (for example, 'fully transformed $\mathrm{MoS}_{2}$ ) gradually raised the peak flux, until saturation was reached with 40 p.p.m. of $\mathrm{MoS}_{2}$, yielding a peak flux of $620 \mathrm{nmol} \mathrm{min}^{-1}$. With the latter reaction, 40 p.p.m. of $\mathrm{MoS}_{2}$ raised overall yield to $30 \mu \mathrm{mol}$ in the first $100 \mathrm{~min}$, giving a typical activity of $18 \mu \mathrm{molh}^{-1}$, which is four times higher than the autocatalytic EY dye under the same conditions ${ }^{43}$. In both cases, addition of small amounts of $\mathrm{HCl}$ regenerates a stopped reaction, suggesting reaction termination is due to proton depletion (Supplementary Fig. 6).

Given the boosted HER performance, it reasons that in the presence of fully transformed $\mathrm{MoS}_{2}$ a preferential charge transfer occurred between the radical $\mathrm{EY}^{-}$and the $\mathrm{MoS}_{2}$. Indeed, with the reductive potential of $\mathrm{EY}$ at $-0.8 \mathrm{~V}$ versus normal hydrogen electrode (NHE), and $\mathrm{MoS}_{2}$ 's conduction band situated at $0.2 \mathrm{~V}$ versus NHE, such a transfer is feasible ${ }^{48,49}$. To support this, the charge transfer was examined using fluorescence quenching correlations between EY and the Li-exfoliated $\mathrm{MoS}_{2}$ (Fig. 4c). It can be seen that the fluorescence quenching interactions between EY and $\mathrm{MoS}_{2}$ are predictive of the eventual $\mathrm{H}_{2}$ yield, with both curves rising to saturation in the presence of 40 p.p.m. of $\mathrm{MoS}_{2}$. The fluorescence quenching following a saturation reaction fitting, vis-à-vis a continual diminishing of fluorescence with increasing quencher concentration (for example, collisional quenching) indicates the formation of a ground-state complex between EY and lithium-exfoliated $\mathrm{MoS}_{2}$, facilitating effectual charge transfer. To further elucidate these interactions, we monitored the excited state interactions via fluorescence decay. As seen in Fig. 4d, the fluorescence lifetime of a singlet excited $\mathrm{EY}$, in the absence of secondary interactions, is $\sim 1 \mathrm{~ns}$. However, interactions with reductive quenchers, such as TEOA, via collisional quenching results in fluorescence lifetime shortening that fits a two exponential decay (Fig. 4e $)^{50}$. Interactions between $\mathrm{EY}$ and $\mathrm{MoS}_{2}$ did not follow this behavior. Instead, the lifetime of EY was maintained at $\sim 1 \mathrm{~ns}$ (Fig. $4 \mathrm{f}$ ), while the fluorescence intensity was attenuated by $75 \%$ at saturation. This indicates adsorption of EY onto $\mathrm{MoS}_{2}$ to form a non-fluorescent groundstate complex that facilitates charge transfer within the complex with an excited state lifetime following the behavior of the $25 \%$ minority free dyes ${ }^{50}$.

As the above were performed with the fully transformed $1 \mathrm{~T}^{\prime}$, effect of partial $1 \mathrm{~T}^{\prime}$ transformation was then investigated. Here, two additional $\mathrm{MoS}_{2}$ batches with diminishing $1 \mathrm{~T}^{\prime}$ 

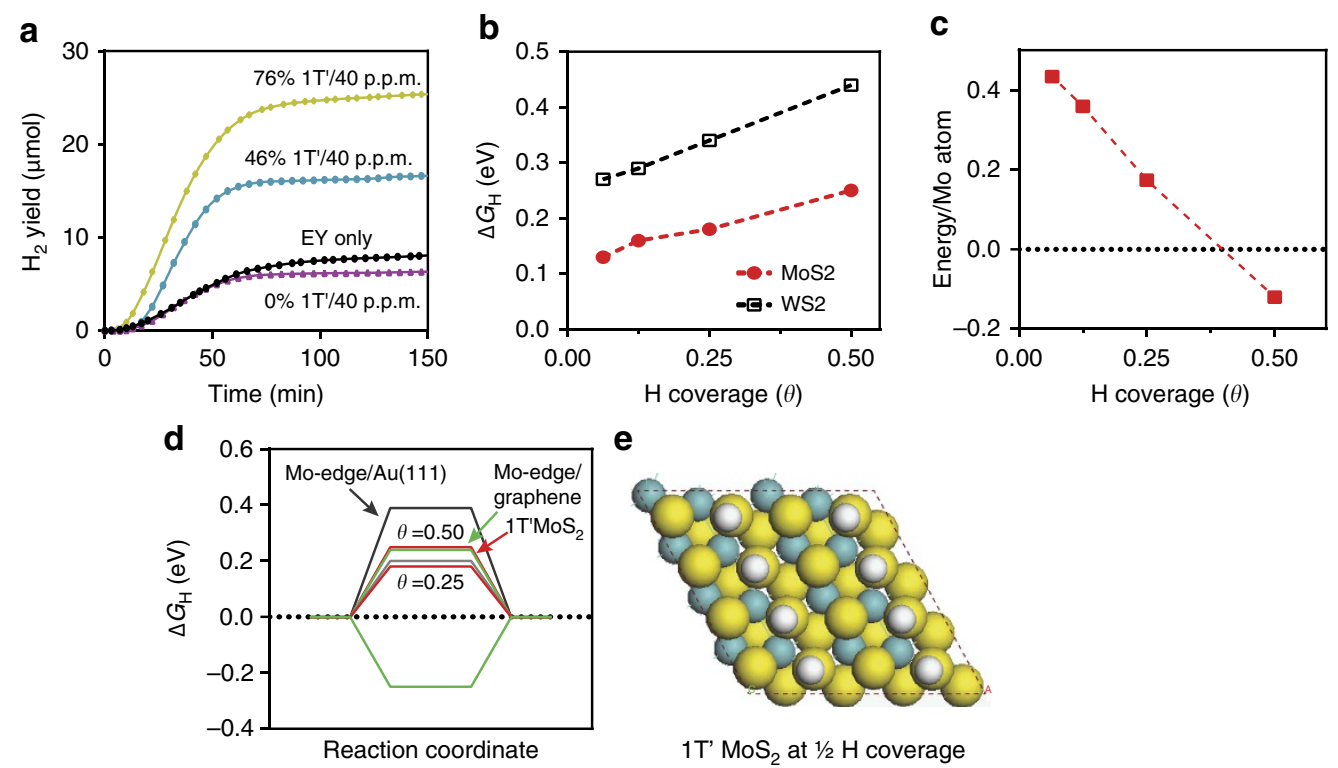

e

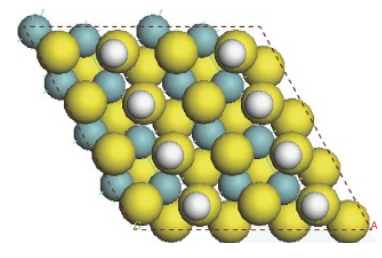

$1 \mathrm{~T}^{\prime} \mathrm{MoS}_{2}$ at $1 / 2 \mathrm{H}$ coverage

Figure 5 | Energetics of Hydrogen Evolution. (a) Cumulative $\mathrm{H}_{2}$ yield with $\mathrm{MoS}_{2}$ undergoing differing degrees of $1 \mathrm{~T}^{\prime}$ transformation. (b) DFT calculated Gibbs free energy of hydrogen adsorption $\left(\Delta G_{H}\right)$ as a function of $\mathrm{H}$ coverage. (c) DFT calculated stability of the MoS $\mathrm{S}_{2}$ structures per Mo atoms as a function of $\mathrm{H}$ coverage. As the $\mathrm{H}$ coverage exceeds 0.4 , the stability of the $1 \mathrm{~T}^{\prime}$ phase (red dashes) surpasses that of the $2 \mathrm{H}$ (black dots). (d) Free-energy diagram of

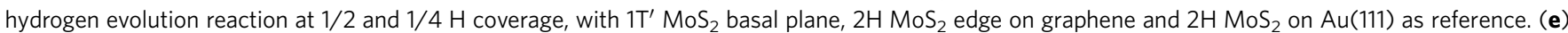
Structural representation of $1 \mathrm{~T}^{\prime}$ at $1 / 2 \mathrm{H}$ coverage.

transformation were used in identical reactions. As shown in Fig. 5a, the $\mathrm{MoS}_{2}$ batches with diminished $1 \mathrm{~T}^{\prime}$ transformation yielded significantly less $\mathrm{H}_{2}$. Indeed, it can be seen that $\mathrm{H}_{2}$ yield scaled proportionately with $1 \mathrm{~T}^{\prime}$ signatures, which dominates the basal plane of the exfoliated sheets (Supplementary Fig. 7, Supplementary Table 2). Given that $1 \mathrm{~T}^{\prime}$ has shown empirical evidence of improved electrical conductivity, it is therefore possible that the improved catalysis seen with the 'fully transformed $\mathrm{MoS}_{2}$ ' is a consequence of better electron conduction from the $\mathrm{EY}^{-}$charge transfer site to the catalytic sites of $\mathrm{MoS}_{2}$. However, it is also possible that the $1 \mathrm{~T}^{\prime}$ transformation fundamentally alters the catalytic mechanism of the $\mathrm{MoS}_{2}$ sheets, possibly giving additional active sites for catalysis.

To investigate, we calculated, via DFT, the free energy of hydrogen adsorption $\left(\Delta G_{\mathrm{H}}\right)$, for the basal plane of each polymorph. From previous reports, it is known that $\left|\Delta G_{H}\right| \approx 0$ describes an optimal adsorption condition for catalysis ${ }^{10}$. Significant deviation in the exothermic direction $\left(\Delta G_{\mathrm{H}}<0\right)$ can indicate irreversible adsorption, consequently, an efficient catalyst such as the $2 \mathrm{H} \mathrm{MoS}$ edge site, tends towards being slightly endothermic $\left(\Delta G_{\mathrm{H}}>0\right)$. From our calculations, $\Delta G_{\mathrm{H}}$ for the $2 \mathrm{H}$ basal plane is $>+1.6 \mathrm{eV}$ regardless of the $\mathrm{H}$ coverage and the absorption site. As a result, the adsorption of $\mathrm{H}$ on the $2 \mathrm{H}$ basal plane is strongly unfavorable. This is consistent with the $2 \mathrm{H}$ surface being catalytically inert ${ }^{9}$. However, when the basal plane is converted to $1 \mathrm{~T}^{\prime}$, the hydrogen binding energy becomes negative (adsorption occurs). As seen in Fig. 5b, the free energy of adsorption here for $1 \mathrm{~T}^{\prime} \mathrm{MoS}_{2}$ is reasonably close to the optimum value of $\gtrsim 0 \mathrm{eV}$ (ref. 12), emerging at $\Delta \mathrm{G}_{\mathrm{H}}=0.13 \mathrm{eV}$ at $1 / 16$ coverage and growing to $0.25 \mathrm{eV}$ as $\mathrm{H}$ coverage increased to $1 / 2$. This indicates that the $1 \mathrm{~T}^{\prime}$ transformed surface is amenable for catalysis, and indeed, the HER improvements with $1 \mathrm{~T}^{\prime}$ fraction is due to catalytic activation of the $\mathrm{MoS}_{2}$ basal plane. Most interestingly, we also observe adsorption of hydrogen to stabilize the $1 \mathrm{~T}^{\prime}$. Indeed, Fig. $5 \mathrm{c}$ shows that when $\mathrm{H}$ coverage exceeds $0.4,1 \mathrm{~T}^{\prime}$ becomes more stable than $2 \mathrm{H}$. As significant benchmark work with $2 \mathrm{H} \mathrm{MoS}$ was performed with $\mathrm{Au}$ (111) supports $^{9}$, we compare $1 \mathrm{~T}^{\prime}$ with $2 \mathrm{H}$ Mo-edge sites, when supported on gold. It can be seen here that $\left|\Delta G_{\mathrm{H}}\right|$ on the $1 \mathrm{~T}^{\prime}$ basal plane is comparable to the $2 \mathrm{H}$ Mo-edge supported on $\mathrm{Au}$ (111) and graphene at 0.25 coverage and slightly outperforms Mo-edges on $\mathrm{Au}(111)$ at 0.5 coverage (Fig. $5 \mathrm{~d}, \mathrm{e})^{51}$. Above all, the shear increase in surface area availability after exfoliation to monolayers (100-1,000-fold greater than bulk solids $)^{52}$ renders the basal plane improvements even more significant.

As $\mathrm{WS}_{2}$ can undergo analogous phase transformations, its catalytic efficiency was similarly analyzed. We show in Supplementary Fig. 8, the $\mathrm{H}_{2}$ flux with $\mathrm{WS}_{2}$ was a third lower than $\mathrm{MoS}_{2}$. For peak $\mathrm{H}_{2}$ flux, $\mathrm{WS}_{2}$ 's $400 \mathrm{nmolmin}^{-1}$ showed approximately threefold improvement over controls, but weaker than the $750 \mathrm{nmol} \mathrm{min}{ }^{-1}$ observed for $\mathrm{MoS}_{2}$. Similarly, $\mathrm{H}_{2}$ yield with $\mathrm{WS}_{2}$ was $18 \mu \mathrm{mol}$ at $t=100 \mathrm{~min}$, which is $33 \%$ lower than $\mathrm{MoS}_{2}$. As fluorescence lifetime measurements revealed an analogous molecular charge transfer pathway, the reduced HER efficiency was thus indicative of $\mathrm{WS}_{2}$ being a less efficient catalyst. From Fig. $5 \mathrm{~b}$, it can be seen that the $\Delta G_{\mathrm{H}}$ of $1 \mathrm{~T}^{\prime}$ transformed $\mathrm{WS}_{2}$ was 0.15 to $0.2 \mathrm{eV}$ higher than $\mathrm{MoS}_{2}$. Nevertheless, this value represents a 10 -fold reduction over $\Delta G_{\mathrm{H}}$ of $2 \mathrm{H} \mathrm{WS}$, indicating catalytic activation of the basal plane comparable to $\mathrm{MoS}_{2}$.

\section{Discussion}

We investigated the structure of $\mathrm{MoS}_{2}$ exfoliated with lithiation intercalation and directly correlated the varying physical structures to the catalytic origin for HER. Particularly, we demonstrated via abberation-corrected scanning transmission electron microscopy that modulated lithiation can lead to complete $1 \mathrm{~T}^{\prime}$ transformation in the basal plane, and corroborated the experimental findings with DFT calculated phase stabilities. It was shown that the Mo atoms of $\mathrm{MoS}_{2}$ became asymmetrically spaced with increased lithiation, thus forming the $1 \mathrm{~T}^{\prime}$ phase. In DFT, the $1 \mathrm{~T}^{\prime}$ was shown to be energetically favorable compared to other possible polymorphs. Nevertheless, external 
perturbations within the environment, including electron imaging parameters, can induce a reversion to $2 \mathrm{H}$.

With regard to catalysis, we have shown that the $1 \mathrm{~T}^{\prime}$ transformation rendered the normally inert basal plane amenable towards hydrogen adsorption and $\mathrm{H}_{2}$ evolution. Indeed, $\Delta \mathrm{G}_{\mathrm{H}}$ on the basal plane of $1 \mathrm{~T}^{\prime} \mathrm{MoS}_{2}$ showed catalytic activation and was lowered from $+1.6 \mathrm{eV}$ in the $2 \mathrm{H}$ to $+0.18 \mathrm{eV}$. Moreover, when $\mathrm{H}$ coverage became $>0.4$, DFT showed $1 \mathrm{~T}^{\prime}$ phase stability surpasses that of $2 \mathrm{H}$. To put this in perspective, the $\Delta \mathrm{G}_{\mathrm{H}}$ of $0.18 \mathrm{eV}$ is comparable to $2 \mathrm{H} \mathrm{MoS}_{2}$ edges on $\mathrm{Au}(111)$, one of the most active HER catalysts yet characterized. This makes the $1 \mathrm{~T}^{\prime}$ $\mathrm{MoS}_{2}$ a state-of-the-art catalyst. In addition, as exfoliation to monolayers increases the surface area by as much as 1,000fold ${ }^{52,53}$, the basal plane activation provides non-trivial increases in catalytic efficiency compared with the edge only catalysis of the $2 \mathrm{H}$ (refs 9,15). We demonstrate this by $\mathrm{H}_{2}$ evolution studies in basic solution ( $\mathrm{pH} 11)$, via self-assembled photocatalytic charge transfer complexes for HER. Here it can be seen that $\mathrm{MoS}_{2}$ catalysts can boost $\mathrm{H}_{2}$ yield of the HER catalytic dye, EY, by fourfold. Moreover, $\mathrm{MoS}_{2}$ itself does not fatigue over the course of the reaction. A clearer understanding of these materials, and the underlying relationship between structure, properties and performance, provides a pathway towards quantitative engineering of $\mathrm{MX}_{2}$ to enable the emerging 'green energy' economy.

\section{Methods}

Lithium intercalation and exfoliation. For $\mathrm{MoS}_{2}$, lithium intercalation was accomplished by immersing $1 \mathrm{~g}$ of $\mathrm{MoS}_{2}$ powder in $10 \mathrm{ml}$ of $1 \mathrm{M} n$-butyl lithium. The mixture was stirred vigorously in an inert atmosphere glovebox for 3 to 10 days. After, the compound was washed over three layers of Whatman filter paper (\#51, ashless) with $200 \mathrm{ml}$ of hexane and then collected in a bottle. Three hundred millilitres of $\mathrm{H}_{2} \mathrm{O}$ was then added, and the mixture was sonicated for $3 \mathrm{~m}$ to extricate the intercalated $\mathrm{MoS}_{2}$ powder from the filter paper. The filter paper was then removed from the bottle, and the solution was sonicated for an additional $1.5 \mathrm{~h}$. Unexfoliated portions were removed with centrifugation at $100 \mathrm{~g}$ for $3 \mathrm{~min}$. The supernatant was then collected, and purified by centrifugation and washing with $\mathrm{H}_{2} \mathrm{O}(3 \times 10,000 \mathrm{~g}$ for $1.5 \mathrm{~h}$, followed by resuspension in water after each centrifugation cycle). The solution was then transferred in to a dialysis bag (Fisher Scientific, MW 5,000), and dialysed against running water for 3 days. The solution was then again centrifuged at $500 \mathrm{~g}$ for $15 \mathrm{~m}$ to remove aggregates, and the resultant samples were used as is. For $\mathrm{WS}_{2}, \mathrm{Li}$ intercalation was performed at $100{ }^{\circ} \mathrm{C}$ in a Parr bomb. Exfoliation and purification of $\mathrm{WS}_{2}$ was the same as $\mathrm{MoS}_{2}$. Atomic force microscopy images of exfoliates sheets are shown in Supplementary Fig. 9.

STEM microscopy and analysis. A FEI Titan G2 80-200 S/TEM with a Cs probe corrector operated at $200 \mathrm{kV}$ was used in this study. High-angle annular dark-field (HAADF) S/TEM images were acquired with an electron probe size of $0.8 \AA$, convergence angle of $18.1 \mathrm{mrad}$, and current of $\sim 100 \mathrm{pA}$ with an annular detector with a collection range of $60-160 \mathrm{mrad}$. The high resolution HAADF images were typically taken at $1,800 \mathrm{k}$ magnification, yielding a pixel size of about $0.23 \AA$ or a frame size of $48 \times 48 \mathrm{~nm}$ for $2,048 \times 2,048$ pixels per frame. Such conditions gave rise to an equivalent electron dose rate of $\sim 2,800$ electrons $\AA^{-2} s^{-1}$. Acquisition of the HAADF image (frame of 2,048 $\times 2,048$ pixels) took $\sim 40 \mathrm{~s}$ at a dwell time of $\sim 10 \mu$ s/pixel. In sub-figures of Figs 1, 2 and 3, the filtered images were obtained by Fast Fourier transformation (FFT) of the HAADF images into reciprocal space, forming FFT patterns. Reciprocal spots from the patterns (typically $\{100\}$ type reflections) were selected, masked with a 60 pixels filter, and then transformed into real space with inverse FFT. Gatan Digital Micrograph was used for the image processing. Additional details on TEM imaging and simulation are shown in Supplementary Methods

Computational details. All density functional calculations were carried out using the Vienna $a b$ initio simulation package ${ }^{54}$ with plane wave basis set and projector augmented-wave pseudopotentials ${ }^{55}$. All energies were calculated with PerdewBurke-Ernzerhof exchange-correlation potentials ${ }^{56}$. For selected structures, we applied the hybrid HSE06 functional ${ }^{57}$ to calculate the electronic band gap. The $\mathrm{MoX}_{2}(\mathrm{X}=\mathrm{Mo}, \mathrm{W})$ monolayers were modeled using surface supercells separated in the periodic direction by a $20 \AA$-thick vacuum slab. We applied a plane wave energy cutoff of $600 \mathrm{eV}$ and $\Gamma$-centered $24 \times 24 \times 1,12 \times 24 \times 1,12 \times 12 \times 1$, and $6 \times 6 \times 1 \mathrm{k}$-points grids for Brillouin zone sampling of the $1 \times 1$ unit cell $(2 \mathrm{H}$ and $1 \mathrm{~T})$, and the $2 \times 1\left(1 \mathrm{~T}^{\prime}\right), 2 \times 2\left(1 \mathrm{~T}^{\prime \prime}\right)$, and $4 \times 4\left(\mathrm{H} / \mathrm{XS}_{2}\right)$ supercells, respectively. The criteria of convergence for energy and force were set as $10^{-4} \mathrm{eV}$ and
$3 \times 10^{-3} \mathrm{eV} / \AA$. The hydrogen adsorption energies were calculated using the $4 \times 4$ surface supercell containing $16 \mathrm{Mo}$ atoms and $32 \mathrm{~S}$ atoms. A dipole correction was applied to cancel the electrostatic interaction between the periodic slabs. The DFT binding energies were calculated as $\Delta E_{\mathrm{H}}=\frac{1}{n}\left(E(\operatorname{surf}+n \mathrm{H})-E(\operatorname{surf})-\frac{n}{2} E\left(\mathrm{H}_{2}\right)\right)$, where $\mathrm{E}($ surf $+\mathrm{nH}), \mathrm{E}$ (surf), and $\mathrm{E}\left(\mathrm{H}_{2}\right)$ are the total energies for the $\mathrm{MoS}_{2}$ surface with $n$ hydrogen atoms adsorbed, the clean $\mathrm{MoS}_{2}$ surface, and the molecular hydrogen in the gas phase, respectively. The most stable $\mathrm{H}$ binding site in the basal plane of $\mathrm{MoS}_{2}$ is on the top of the $\mathrm{S}$ atoms. We define $\mathrm{H}$ coverage as the ratio of the number of $\mathrm{H}$ and $\mathrm{Mo}$ atoms in the basal plane. For $1 \mathrm{~T}^{\prime} \mathrm{MoS}_{2}$, we obtained binding energies of $-0.16 \mathrm{eV},-0.13 \mathrm{eV}$, and $-0.11 \mathrm{eV}$, and $-0.035 \mathrm{eV}$ at the $1 / 16 \mathrm{H}$, $1 / 8 \mathrm{H}, 1 / 4 \mathrm{H}$, and $1 / 2 \mathrm{H}$ coverage. For the $4 \times 4$ supercell, this corresponds to $n=1$, 2,4 , and 8 , respectively. The adsorption free energy was calculated by adding a thermal correction to the binding energy $\Delta G_{\mathrm{H}}=\Delta E_{\mathrm{H}}+\Delta E_{\mathrm{ZPE}}-T \Delta S_{\mathrm{H}}$, where $\Delta E_{\mathrm{ZPE}}$ and $T \Delta S_{\mathrm{H}}$ are, respectively, the differences in the zero point energy and entropy contribution between the $\mathrm{H}$ adsorbed state and $\mathrm{H}_{2}$ in the gas phase. We took $\Delta \mathrm{S}_{\mathrm{H}}=-1 / 2 \mathrm{~S}\left(\mathrm{H}_{2}\right)$, where $1 / 2 \mathrm{~S}\left(\mathrm{H}_{2}\right)$ is the entropy of $1 / 2 \mathrm{H}_{2}$ in the gas phase at standard conditions $(T=298.15 \mathrm{~K}$, Pressure $=1 \mathrm{~atm})$. We used the assumption that the vibrational entropy in the adsorbed state is small ${ }^{58}$. For $\mathrm{H} / 1 \mathrm{~T}^{\prime} \mathrm{MoS}_{2}$, $E_{\mathrm{ZPE}}=0.228 \mathrm{eV}$ (vibrational frequencies $2,530.1 \mathrm{~cm}^{-1}, 637.6 \mathrm{~cm}^{-1}, 519.8 \mathrm{~cm}^{-1}$ ) and $E_{\mathrm{ZPE}}=0.271 \mathrm{eV}$ for $\mathrm{H}_{2}$. With these values, the Gibbs free energy was calculated as $\Delta G_{\mathrm{H}}=\Delta E_{\mathrm{H}}+0.29 \mathrm{eV}$. Additional details are available in Supplementary Methods.

Photocatalysis. Stock mixtures of $30 \% \mathrm{v} / \mathrm{v}$ TEOA and $10 \mathrm{mg} \mathrm{ml}^{-1}$ Eosin Y were freshly prepared for each reaction. $\mathrm{MoS}_{2}\left(\right.$ and $\mathrm{WS}_{2}$ ) were prepared at twice the desired final concentration (for example, 80 p.p.m.). To prepare the reaction mixture, $35 \mathrm{ml}$ of the $30 \% \mathrm{v} / \mathrm{v}$ TEOA was mixed with $35 \mathrm{ml}$ of the $\mathrm{MoS}_{2}$ or $\mathrm{WS}_{2}$, reaching the final concentration of $15 \% \mathrm{v} / \mathrm{v}$ TEOA and the predetermined $\mathrm{MoS}_{2} / \mathrm{WS}_{2}$ concentration (for example, 40 p.p.m.). The mixture was then transferred to a $250 \mathrm{ml}$ two-neck flask, to which $1 \mathrm{ml}$ of the $10 \mathrm{mg} \mathrm{ml}^{-1}$ Eosin Y was added. The flask was then covered in aluminum foil, stirred and purged with continuous Ar flow (20 c.c.m.) for 20 min. After Ar purge, the aluminum foil covering was removed, and the mixture was illuminated at 1 sun. With the Ar flow kept at 20 c.c.m., gas samples were continuously monitored at $90 \mathrm{~s}$ intervals using an Inficon 3,000 micro GC gas analyzer until reaction termination.

\section{References}

1. Bard, A. J. \& Fox, M. A. Artificial photosynthesis: solar splitting of water to hydrogen and oxygen. Acc. Chem. Res. 28, 141-145 (1995).

2. Lewis, N. S. \& Nocera, D. G. Powering the planet: Chemical challenges in solar energy utilization. Proc. Natl Acad. Sci. USA 103, 15729-15735 (2006).

3. Turner, J. A. Sustainable hydrogen production. Science 305, 972-974 (2004).

4. Walter, M. G. et al. Solar water splitting cells. Chem. Rev. 110, 6446-6473 (2010).

5. Colbeau, A. \& Vignais, P. M. The membrane-bound hydrogenase of Rhodopseudomonas capsulata: Stability and catalytic properties. Biochim. Biophys. Acta Enzymol. 662, 271-284 (1981).

6. Conway, B. E. \& Tilak, B. V. Interfacial processes involving electrocatalytic evolution and oxidation of $\mathrm{H}_{2}$, and the role of chemisorbed $\mathrm{H}$. Electrochim. Acta 47, 3571-3594 (2002).

7. Tafel, J. Ueber strychnin. Justus Liebigs Annalen der Chemie. 301, 285-348 (1898).

8. Tafel, J. \& Naumann, K. Die elektrolytische Reduction des Strychnis und Brucins. Berichte der deutschen chemischen. Gesellschaft. 34, 3291-3299 (1901).

9. Jaramillo, T. F. et al. Identification of active edge sites for electrochemical $\mathrm{H}_{2}$ evolution from $\mathrm{MoS}_{2}$ nanocatalysts. Science 317, 100-102 (2007).

10. Bonde, J., Moses, P. G., Jaramillo, T. F., Norskov, J. K. \& Chorkendorff, I. Hydrogen evolution on nano-particulate transition metal sulfides. Faraday Discuss. 140, 219-231 (2009).

11. Raybaud, P., Hafner, J., Kresse, G., Kasztelan, S. \& Toulhoat, H. Ab initio study of the $\mathrm{H}_{2}-\mathrm{H}_{2} \mathrm{~S} / \mathrm{MoS}_{2}$ gas-solid interface: the nature of the catalytically active sites. J. Catal. 189, 129-146 (2000).

12. Hinnemann, B. et al. Biomimetic hydrogen evolution: $\mathrm{MoS}_{2}$ nanoparticles as catalyst for hydrogen evolution. J. Am. Chem. Soc. 127, 5308-5309 (2005).

13. Kong, D. et al. Synthesis of $\mathrm{MoS}_{2}$ and $\mathrm{MoSe}_{2}$ films with vertically aligned layers. Nano Lett. 13, 1341-1347 (2013).

14. Karunadasa, H. I. et al. A molecular $\mathrm{MoS}_{2}$ edge site mimic for catalytic hydrogen generation. Science 335, 698-702 (2012).

15. Kibsgaard, J., Chen, Z., Reinecke, B. N. \& Jaramillo, T. F. Engineering the surface structure of $\mathrm{MoS}_{2}$ to preferentially expose active edge sites for electrocatalysis. Nat. Mater. 11, 963-969 (2012).

16. Kibsgaard, J., Jaramillo, T. F. \& Besenbacher, F. Building an appropriate active-site motif into a hydrogen-evolution catalyst with thiomolybdate $\left[\mathrm{Mo}_{3} \mathrm{~S}_{13}\right]_{2}$ - clusters. Nat. Chem. 6, 248-253 (2014).

17. Kim, J., Byun, S., Smith, A. J., Yu, J. \& Huang, J. Enhanced electrocatalytic properties of transition-metal dichalcogenides sheets by spontaneous gold nanoparticle decoration. J. Phys. Chem. Lett. 4, 1227-1232 (2013). 
18. Lukowski, M. A. et al. Enhanced hydrogen evolution catalysis from chemically exfoliated metallic $\mathrm{MoS}_{2}$ nanosheets. J. Am. Chem. Soc. 135, 10274-10277 (2013).

19. Voiry, D. et al. Conducting $\mathrm{MoS}_{2}$ nanosheets as catalysts for hydrogen evolution reaction. Nano Lett. 13, 6222-6227 (2013).

20. Azizi, A. et al. Dislocation motion and grain boundary migration in two-dimensional tungsten disulphide. Nat. Commun. 5, 4867 (2014).

21. Erickson, K. et al. Determination of the local chemical structure of graphene oxide and reduced graphene oxide. Adv. Mater. 22, 4467-4472 (2010).

22. Gómez-Navarro, C. et al. Atomic structure of reduced graphene oxide. Nano Lett. 10, 1144-1148 (2010).

23. Somoano, R. B., Hadek, V. \& Rembaum, A. Alkali metal intercalates of molybdenum disulfide. J. Chem. Phys. 58, 697-701 (1973).

24. Py, M. A. \& Haering, R. R. Structural destabilization induced by lithium intercalation in $\mathrm{MoS}_{2}$ and related compounds. Can. J. Phys. 61, 76-84 (1983).

25. Chrissafis, K. et al. Structural studies of $\mathrm{MoS}_{2}$ intercalated by lithium. Mater. Sci. Eng. B 3, 145-151 (1989)

26. Jiménez Sandoval, S., Yang, D., Frindt, R. F. \& Irwin, J. C. Raman study and lattice dynamics of single molecular layers of $\mathrm{MoS}_{2}$. Phys. Rev. B 44, 3955-3962 (1991).

27. Qin, X. R., Yang, D., Frindt, R. F. \& Irwin, J. C. Real-space imaging of single-layer $\mathrm{MoS}_{2}$ by scanning tunneling microscopy. Phys. Rev. B 44, 3490-3493 (1991)

28. Schumacher, A., Scandella, L., Kruse, N. \& Prins, R. Single-layer $\mathrm{MoS}_{2}$ on mica: studies by means of scanning force microscopy. Surf. Sci. 289, L595-L598 (1993).

29. Dungey, K. E., Curtis, M. D. \& Penner-Hahn, J. E. Structural characterization and thermal stability of $\mathrm{MoS}_{2}$ intercalation compounds. Chem. Mater. 10, 2152-2161 (1998)

30. Heising, J. \& Kanatzidis, M. G. Structure of restacked $\mathrm{MoS}_{2}$ and $\mathrm{WS}_{2}$ elucidated by electron crystallography. J. Am. Chem. Soc. 121, 638-643 (1999).

31. Petkov, V. et al. Structure of nanocrystalline materials using atomic pair distribution function analysis: Study of $\mathrm{LiMoS}_{2}$. Phys. Rev. B 65, 092105 (2002).

32. Maitra, U. et al. Highly effective visible-light-induced $\mathrm{H}_{2}$ generation by singlelayer $1 \mathrm{~T}-\mathrm{MoS}_{2}$ and a nanocomposite of few-layer $2 \mathrm{H}-\mathrm{MoS}_{2}$ with heavily nitrogenated graphene. Angew Chem. Int. Ed. 52, 13057-13061 (2013)

33. Eda, G. et al. Photoluminescence from chemically exfoliated $\mathrm{MoS}_{2}$. Nano Lett. 11, 5111-5116 (2011)

34. Eda, G. et al. Coherent atomic and electronic heterostructures of single-layer $\mathrm{MoS}_{2}$. ACS Nano 6, 7311-7317 (2012).

35. Kappera, R. et al. Phase-engineered low-resistance contacts for ultrathin $\mathrm{MoS}_{2}$ transistors. Nat. Mater. 13, 1128-1134 (2014).

36. Asadi, M. et al. Robust carbon dioxide reduction on molybdenum disulphide edges. Nat. Commun. 5, 4470 (2014).

37. Voiry, D. et al. Enhanced catalytic activity in strained chemically exfoliated $\mathrm{WS}_{2}$ nanosheets for hydrogen evolution. Nat. Mater. 12, 850-855 (2013).

38. Calandra, M. Chemically exfoliated single-layer $\mathrm{MoS}_{2}$ : Stability, lattice dynamics, and catalytic adsorption from first principles. Phys. Rev. B 88, 245428 (2013)

39. Tung, V. C., Allen, M. J., Yang, Y. \& Kaner, R. B. High-throughput solution processing of large-scale graphene. Nat. Nanotechnol. 4, 25-29 (2009).

40. Tung, V. C. et al. Low-temperature solution processing of graphene-carbon nanotube hybrid materials for high-performance transparent conductors. Nano Lett. 9, 1949-1955 (2009).

41. Tung, V. C. et al. Towards solution processed all-carbon solar cells: a perspective. Energy Environ. Sci. 5, 7810-7818 (2012).

42. Heising, J. \& Kanatzidis, M. G. Exfoliated and restacked $\mathrm{MoS}_{2}$ and $\mathrm{WS}_{2}$ : Ionic or neutral species? Encapsulation and ordering of hard electropositive cations. J. Am. Chem. Soc. 121, 11720-11732 (1999).

43. Du, P., Schneider, J., Jarosz, P. \& Eisenberg, R. Photocatalytic generation of hydrogen from water using a platinum(II) terpyridyl acetylide chromophore. J. Am. Chem. Soc. 128, 7726-7727 (2006).

44. Dempsey, J. L., Brunschwig, B. S., Winkler, J. R. \& Gray, H. B. Hydrogen evolution catalyzed by cobaloximes. Acc. Chem. Res. 42, 1995-2004 (2009).

45. Min, S. \& Lu, G. Enhanced electron transfer from the excited Eosin Y to mpg- $\mathrm{C}_{3} \mathrm{~N}_{4}$ for highly efficient hydrogen evolution under $550 \mathrm{~nm}$ irradiation. J. Phys. Chem. C 116, 19644-19652 (2012).

46. Min, S. \& Lu, G. Sites for high efficient photocatalytic hydrogen evolution on a limited-layered $\mathrm{MoS}_{2}$ cocatalyst confined on graphene sheets-the role of graphene. J. Phys. Chem. C 116, 25415-25424 (2012).

47. Lazarides, T. et al. Making hydrogen from water using a homogeneous system without noble metals. J. Am. Chem. Soc. 131, 9192-9194 (2009).
48. Schneemeyer, L. F. \& Wrighton, M. S. Flat-band potential of n-type semiconducting molybdenum disulfide by cyclic voltammetry of two-electron reductants: interface energetics and the sustained photooxidation of chloride. J. Am. Chem. Soc. 101, 6496-6500 (1979).

49. Min, S. \& Lu, G. Dye-sensitized reduced graphene oxide photocatalysts for highly efficient visible-light-driven water reduction. J. Am. Chem. Soc. 115, 13938-13945 (2011).

50. Valeur, B. Molecular Fluorescence: Principles and Applications (WILEY-VCH, 2001).

51. Tsai, C., Abild-Pedersen, F. \& Nørskov, J. K. Tuning the $\mathrm{MoS}_{2}$ edge-site activity for hydrogen evolution via support interactions. Nano Lett. 14, 1381-1387 (2014).

52. Sanchez, V. C., Jachak, A., Hurt, R. H. \& Kane, A. B. Biological interactions of graphene-family nanomaterials: an interdisciplinary review. Chem. Res. Toxicol. 25, 15-34 (2011).

53. Chou, S. S. et al. Chemically exfoliated $\mathrm{MoS}_{2}$ as near-infrared photothermal agents. Angew Chem. Int. Ed. 52, 4160-4164 (2013).

54. Kresse, G. \& Furthmüller, J. Efficient iterative schemes for ab initio totalenergy calculations using a plane-wave basis set. Phys. Rev. B 54, 11169-11186 (1996).

55. Blöchl, P. E. Projector augmented-wave method. Phys. Rev. B 50, 17953-17979 (1994).

56. Perdew, J. P., Burke, K. \& Ernzerhof, M. Generalized gradient approximation made simple. Phys. Rev. Lett. 77, 3865-3868 (1996).

57. Heyd, J., Scuseria, G. E. \& Ernzerhof, M. Hybrid functionals based on screened Coulomb potential. J. Chem. Phys. 118, 8207-8215 (2003).

58. Nørskov, J. K. et al. Trends in the exchange current for hydrogen evolution. J. Electrochem. Soc. 152, J23-J26 (2005).

\section{Acknowledgements}

This work was supported by the U.S. Department of Energy, Office of Science, Office of Basic Energy Sciences, Division of Materials Sciences and Engineering. XPS and gas chromatography experiments were supported by the U.S. Department of Energy, Office of Science, Office of Basic Energy Sciences, Catalysis Science Program grant DE-FG0202ER15368. Fluorescence decay measurements were performed at the Center for Integrated Nanotechnologies, a U.S. Department of Energy, Office of Basic Energy Sciences user facility. Computing resources were provided by the National Energy Research Scientific Computing Center and the Texas Advanced Computing Center (TACC). N.S. was partially supported with funding Sandia National Laboratories. We thank Ana B. Trujillo and James A. Ohlhausen for AFM use. Sandia National Laboratories is a multiprogram laboratory managed and operated by Sandia Corporation, a wholly owned subsidiary of Lockheed Martin Corporation, for the U.S. Department of Energy's National Nuclear Security Administration under contract DE-AC04-94AL85000.

\section{Author contributions}

The study concept and design was provided by S.C., N.S. and C.J.B. Materials synthesis was performed by S.C. Catalysis experiments were performed by S.C. and E.C. Spectroscopy experiments were performed by S.L., T.L., K.A., B.K. and S.C. DFT calculations were performed by N.S. STEM imaging was performed by P.L. with S.C STEM simulations were performed by P.L. All authors discussed the results and contributed to the analysis of the data. Critical revision of the article for intellectual content was conducted by all authors.

\section{Additional information}

Supplementary Information accompanies this paper at http://www.nature.com/ naturecommunications

Competing financial interests: The authors declare no competing financial interests

Reprints and permission information is available online at http://npg.nature.com/ reprintsandpermissions/

How to cite this article: Chou, S. S. et al. Understanding catalysis in a multiphasic two-dimensional transition metal dichalcogenide. Nat. Commun. 6:8311 doi: $10.1038 /$ ncomms9311 (2015)

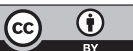

This work is licensed under a Creative Commons Attribution 4.0 International License. The images or other third party material in this article are included in the article's Creative Commons license, unless indicated otherwise in the credit line; if the material is not included under the Creative Commons license, users will need to obtain permission from the license holder to reproduce the material. To view a copy of this license, visit http://creativecommons.org/licenses/by/4.0/ 